\title{
Células Cebadas en Pulmón y Nervio Periférico en la Intoxicación Crónica con Karwinskia humboldtiana en Rata Wistar: Estudios Histológico e Histoquímico
}

\author{
Lung and Peripheral Nerve Mast Cells in Chronic Intoxication with \\ Karwinskia humboldtiana in Wistar Rat: Histological and Histochemical Studies
}

García, G. R."; Salazar, L. M. E.*; Becerra, V. E. M.*; Romero, D. V.*; García, J. J."; Soto, D. A.* \& Sepúlveda, S. J."

GARCÍA, G. R.; SALAZAR, L. M. E.; BECERRA, V. E. M.; ROMERO, D. V.; GARCÍA, J. J.; SOTO, D. A. \& SEPÚLVEDA, S. J. Células cebadas en pulmón y nervio periférico en la intoxicación crónica con Karwinskia humboldtiana en rata wistar: estudio histológico e histoquímico. Int. J. Morphol., 31(4):1216-1222, 2013.

RESUMEN: Karwinskia humboldtiana (Kh) es un arbusto venenoso responsable de numerosos casos de intoxicación accidental en humanos. En estudios previos en nuestro laboratorio reportamos un incremento de células cebadas en nervio periférico (NP) durante la intoxicación con $\mathrm{Kh}$, este hallazgo no ha sido reportado previamente en otros órganos durante esta intoxicación por lo que en el presente estudio buscamos la presencia de estas células en otros órganos, además de distinguir subpoblaciones de células cebadas mediante reacciones histoquímicas para la identificación de los gránulos de secreción. El objetivo de este estudio fue evaluar la presencia de células cebadas en órganos distintos al NP y diferenciar histoquímicamente la composición de sus gránulos. Se utilizaron 32 ratas Wistar, se dividieron en cuatro grupos $(n=8)$ en donde 5 ratas de cada grupo fueron intoxicadas y 3 fueron control no intoxicadas. A las ratas intoxicadas se les administraron por vía oral 3,5 g/kg del fruto seco y molido de $\mathrm{Kh}$ fraccionados en 5 dosis de 1,5; 0,5, 0,5; 0,5 y 0,5 g/kg los días $0,3,7,10$ y 14 respectivamente. Las ratas control solo recibieron agua. Cada grupo fue sacrificado a diferentes tiempos según la evolución de la parálisis. Se obtuvieron muestras de Hígado, Riñón, Pulmón y SNP, se procesaron hasta obtener bloques de parafina, se obtuvieron cortes y se tiñeron con azul de toluidina, PAS, Azul alciano/PAS y Azul alciano/Safranina. Se identificó la presencia de células cebadas en NP y pulmón con la tinción de azul de toluidina y se realizo un estudio morfométrico observando un incremento progresivo del número de células cebadas por grupo así como variaciones histoquímicas en sus gránulos en cada etapa y órgano analizado, lo que sugiere la participación de las células cebadas y sus secreciones en cada una de las etapas de la intoxicación crónica con el fruto maduro de Kh

PALABRAS CLAVE: Karwinskia humboldtiana; Nervio periférico; Pulmón; Células cebadas.

\section{INTRODUCCIÓN}

Karwinskia humboldtiana $(\mathrm{Kh})$ también conocida como tullidora, capulín tullidór o coyotillo, es un arbusto venenoso de la familia Rhamnaceae, se distribuye a lo largo de la república Mexicana, sur de Estados Unidos, América central y norte de Colombia (Fernández, 1992). Dreyer et al. (1975) aislaron del endocarpio de Kh cuatro antracenonas diméricas, que se ha demostrado que son las principales responsables de la toxicidad, denominadas por su peso molecular, T-544, T-516, T-496 y T-514 o peroxisomicina A1 (PA1).

Se han reportado numerosos casos accidentales en humanos de intoxicación por Kh (Castillo et al., 1920; Segovia \& Zermeño, 1972; Bustamante-Sarabia et al., 1978; Puértolas et al., 1984; Arellano et al., 1994; Bermúdez et al., 1995; Ocampo-Roosens et al., 2007). El cuadro clínico de esta intoxicación varía de acuerdo a la cantidad del fruto ingerido, ya que si se consume en gran cantidad, el cuadro paralítico no se presenta, solamente síntomas respiratorios y se puede presentar la muerte en 2 o 3 días. Si se consume en pequeñas cantidades, después de algunas semanas aparece un cuadro clínico de parálisis flácida, simétrica, ascendente y progresiva, que puede conducir a la muerte (Segovia \& Zermeño) o, en algunos casos presentar una recuperación lenta del cuadro paralítico (Arellano et al.).

Salazar-Leal et al. (2006), reportaron un modelo de intoxicación crónica con Kh, administrando dosis fraccionadas del fruto maduro de esta planta a la rata Wistar, logrando reproducir los datos clínicos que se presentan en humanos cuando no mueren por esta intoxicación. Así se

\footnotetext{
* Departamento de Histología. Facultad de Medicina. Universidad Autónoma de Nuevo León, Monterrey, Nuevo León, México.

*** Departamento de Farmacología y Toxicología. Facultad de Medicina. Universidad Autónoma de Nuevo León, Monterrey, Nuevo León, México.
} 
GARCÍA, G. R.; SALAZAR, L. M. E.; BECERRA, V. E. M.; ROMERO, D. V.; GARCÍA, J. J.; SOTO, D. A. \& SEPÚLVEDA, S. J. Células cebadas en pulmón y nervio periférico en la intoxicación crónica con Karwinskia humboldtiana en rata wistar: estudio histológico e histoquímico. Int. J. Morphol., 31(4):1216-1222, 2013.

pudieron identificar las siguientes etapas: sin paresia, paresia, parálisis y recuperación de la parálisis. En la evaluación histopatológica se reporto una desmielinización segmentaria del nervio periférico, que fue aumentando en forma progresiva, alteraciones en el trayecto y grosor axonal a partir del grupo sin paresia hasta el de parálisis, presentando una reversión clínica e histopatológica en el grupo de recuperación. En estas muestras se observo un incremento de células mononucleares identificadas en su mayoría como células cebadas en el endoneurio del nervio periférico, sin embargo no existen reportes acerca del número de células ni de las variaciones en el contenido de los granulos secretorios, que pudieran presentarse a lo largo de la intoxicación.

Utilizando este mismo modelo de intoxicación, Becerra-Verdin et al. (2009), reportaron diversas alteraciones morfológicas en las neuronas y tractos de la vía motora del SNC, incluyendo la corteza cerebral y cerebelo. En este estudio no se reportaron infiltrados de células mononucleares en los órganos del SNC analizados.

Actualmente no existen reportes acerca de la existencia de infiltrados de células cebadas en órganos distintos al SNP durante la intoxicación crónica con el fruto de Kh.

Las células cebadas son principalmente conocidas por el papel que juegan en las reacciones anafilácticas (Metcalfe et al., 1997). Se ha reportado que participan en el nervio periférico, tanto en procesos de desmielinizacion como de remielinizacion (Bienestock et al., 1991; Esposito et al., 2002), así como en pulmón en distintos modelos experimentales de intoxicaciones químicas (Takeoka et al., 1962; Kay et al.,1967; Doebler et al., 1992; Jacevic et al., 2007; Taylor et al., 1984; Okano et al., 1985; De-Matos et al., 2001) en donde el numero de este tipo celular se ve incrementado considerablemente.

En el presente estudio evaluamos al nervio periférico, hígado, riñón y pulmón en busca de infiltrados de células cebadas para cuantificarlas y diferenciar la composición histoquímica de sus gránulos en distintas subpoblaciones de células cebadas, que pudieran estar presentes en cada uno de los órganos y etapas evaluadas del modelo de intoxicación crónica con el fruto de Kh.

\section{MATERIAL Y MÉTODO}

Administración de Karwinskia humboldtiana y grupos de estudio. Se utilizaron 32 ratas Wistar, machos y hembras, se distribuyeron en cuatro grupos $(n=8)$ en donde 5 ratas fueron intoxicadas y 3 fueron control no intoxicadas. Los animales se mantuvieron bajo condiciones estándar de laboratorio. Después de un periodo de 6 horas de ayuno, las ratas a intoxicar recibieron por vía oral y por medio de sonda orogástrica, 5 dosis del fruto seco, molido y cribado de $\mathrm{Kh}$. La primera dosis fue de $1,5 \mathrm{~g} / \mathrm{kg}$ (Día 0 ) seguida por 4 dosis de $0,5 \mathrm{~g} / \mathrm{kg}$ los días $3,7,10$ y 14 posteriores a la primera dosis (Dosis total de $3,5 \mathrm{~g} / \mathrm{kg}$ ). Las ratas control solamente recibieron agua. Todas las ratas fueron evaluadas clínicamente. Se determino el peso diariamente durante las primeras dos semanas, cada tercer día de la semana 3 a la 8 y semanalmente a partir de la semana 8 hasta el final del estudio al día 112. La evaluación clínica incluyó la apariencia del pelo, movilidad espontánea, tono muscular, anormalidades de la marcha, frecuencia respiratoria, pérdida de peso, debilidad de extremidades y parálisis.

Recolección de muestras. Las ratas intoxicadas y control fueron sacrificadas por dislocación cervical a diferentes tiempos después del primer día de intoxicación. El primer grupo fue sacrificado previo a la aparición de la paresia en las ratas intoxicadas en el día 24 (Grupo I). El segundo grupo fue sacrificado cuando las ratas intoxicadas presentaban paresia en el día 48 (Grupo II). El tercer grupo correspondió al de las ratas intoxicadas que presentaban parálisis y fue sacrificado al día 58 (Grupo III) y finalmente el grupo IV correspondió al de las ratas intoxicadas que presentaron recuperación clínica de la parálisis y fueron sacrificadas al día 112.

Histología. Se obtuvieron muestras de nervio periférico, hígado, riñón y pulmón, se fijaron por inmersión en una solución de formalina y se procesaron por la técnica histológica de rutina hasta su inclusión en bloques de parafina. La evaluación histológica se realizó en cortes con un grosor de 5 a 7 micras. Todos los cortes fueron teñidos con H\&E.

Histoquímica. En los casos en donde se presentó un incremento de células mononucleares se realizo la tinción con azul de toluidina al 2\% (AT) para la identificación de células cebadas. Para identificar de forma selectiva los granulos de las células cebadas según su reactividad, se llevaron a cabo las reacciones histoquímicas del Acido Periódico de Shiff (PAS) para la identificación de gránulos con mucopolisacaridos, azul alciano/PAS (AA/PAS) para gránulos con mucinas acidas y neutras, y azul alciano / safranina (AA/SAF) para la identificación de gránulos con aminas biogénicas.

\section{Morfometría}

Nervio periférico. Se utilizaron 5 cortes longitudinales de nervio periférico por cada grupo de ratas intoxicadas con $\mathrm{Kh}$ y control. Las muestras se observaron con microscopio óptico a 400x, al cual se le adaptó un ocular cuadriculado 
con un área total de $0,062 \mathrm{~mm} 2$. Se realizó el conteo de células cebadas en las muestras teñidas con AT, y las que mostraron positividad para cada una de las reacciones histoquímicas aplicadas: PAS, AA/PAS y AA/SAF. Los resultados se expresaron en número de células cebadas, o con positividad para cada una de las reacciones histoquímicas por $\mathrm{mm} 2(\mathrm{CC} / \mathrm{mm} 2)$.

Hígado, riñón y pulmón. Se analizaron 5 cortes de cada uno de los órganos de interés: hígado, riñón y pulmón teñidos con AT, y de histoquímica de PAS, AA/PAS, y AA/SAF por cada grupo de ratas intoxicadas con Kh y control. De cada corte se seleccionaron 8 campos al azar, en donde se cuantificaron todas las células observadas, diferenciando a las células cebadas de los demás tipos celulares presentes. Los valores obtenidos se expresaron en porcentaje (\%).

Análisis estadístico. Los valores obtenidos para cada tipo de granulo identificado se contrastaron por medio de la prueba estadística t de student con el control, análisis de varianza
(ANOVA) para la identificación de diferencias de las varianzas inter-grupos y mediante la prueba de Tukey, para identificar de cual o cuales grupos depende las diferencias en las varianzas en caso de presentarse. Se utilizó el programa estadístico SPSS 15.0 con una significancia de $\mathrm{P} \leq 0,05$.

\section{RESULTADOS}

Los cortes de nervio periférico y de pulmón mostraron infiltrados de células cebadas, mientras que los de hígado y riñón solamente presentaron escasas células aisladas.

Nervio periférico . El estudio morfológico del nervio periférico confirmó los hallazgos histopatológicos reportados por Salazar-Leal et al. En estas muestras se observó la presencia de infiltrados mononucleares en el endoneurio compuestos en su mayoría por células cebadas, identificadas por la positividad para la tinción con azul de toluidina (Figs. 1A y 1B).

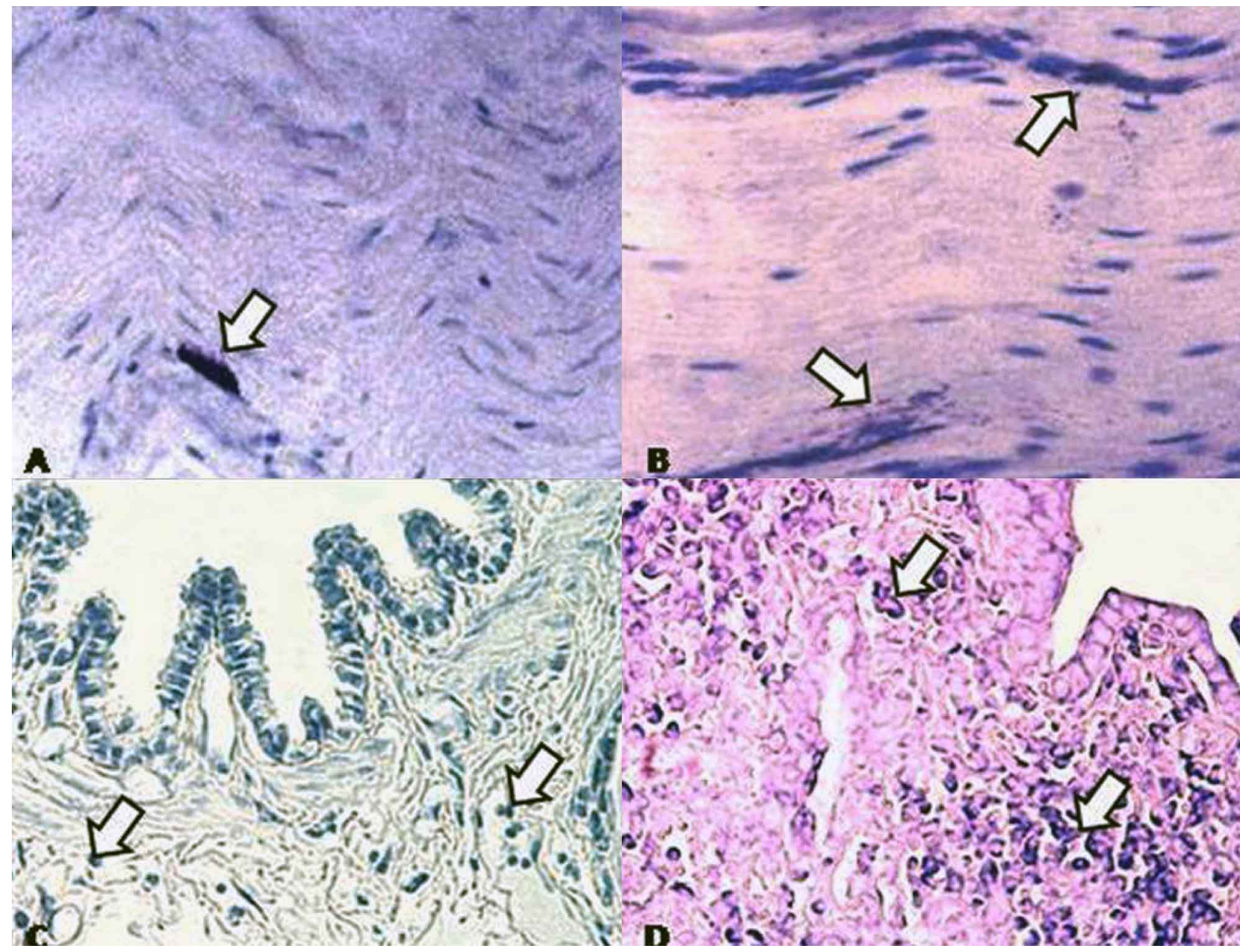

Fig. 1. A. Microfotografía de nervio periférico de rata control que muestra escasas células cebadas en endoneurio (flecha). B. Microfotografía de Nervio periférico de rata intoxicada con Kh que muestra abundantes células cebadas en endoneurio (flechas). C. Microfotografía de pulmón de rata control que muestra un bronquiolo con escasas células cebadas en la lámina propia. D. Microfotografía de pulmón de rata intoxicada con Kh que muestra abundantes células cebadas en la lámina propia (flechas). 
El análisis morfométrico de las células cebadas del nervio periférico, reveló la presencia de células cebadas en las muestras control, y en las muestras experimentales un incremento gradual hasta el grupo III, llegando a su pico máximo en el grupo IV, en donde también se observo un incremento significativo de las células con gránulos PAS positivos en comparación al control. Las células cebadas con gránulos AA/SAF positivos mostraron un decremento significativo en relación al control en los grupos II y III (Fig. 2).

Con la prueba estadística de ANOVA determinamos que en el nervio periférico, durante la intoxicación crónica con Kh, no se presentan diferencias significativas en las varianzas inter-grupos de las células con gránulos positivos a las reacciones de PAS, AA/PAS y AA/SAF razón por la cual se omitió la prueba de Tukey.

Pulmón. El estudio morfológico del pulmón reveló la presencia de grandes acúmulos de células cebadas positivas en la tinción con AT en las regiones peribronquiales, con invasión hacia el epitelio y músculo liso, que en algunos casos modificaban la luz del bronquiolo, perdiéndose el aspecto estrellado de la mucosa, ya que aparecen acumulos en forma de nódulos que protruyen hacia la luz del bronquiolo (Figs. 1C y 1D). Además se observó la presencia de congestión vascular, fibrosis y engrosamientos de los tabiques alveolares a expensas de los acumulos de células cebadas. Estos hallazgos se presentaron en todos los grupos intoxicados con $\mathrm{Kh}$.

El estudio morfométrico de las células cebadas de pulmón teñidas con AT, PAS, AA/PAS y AA/SAF reveló que en los distintos grupos de este modelo de intoxicación, existieron diferencias significativas en cuanto al número de estas células, en comparación a su respectivo control y que estas llegan a su pico máximo durante el grupo IV.

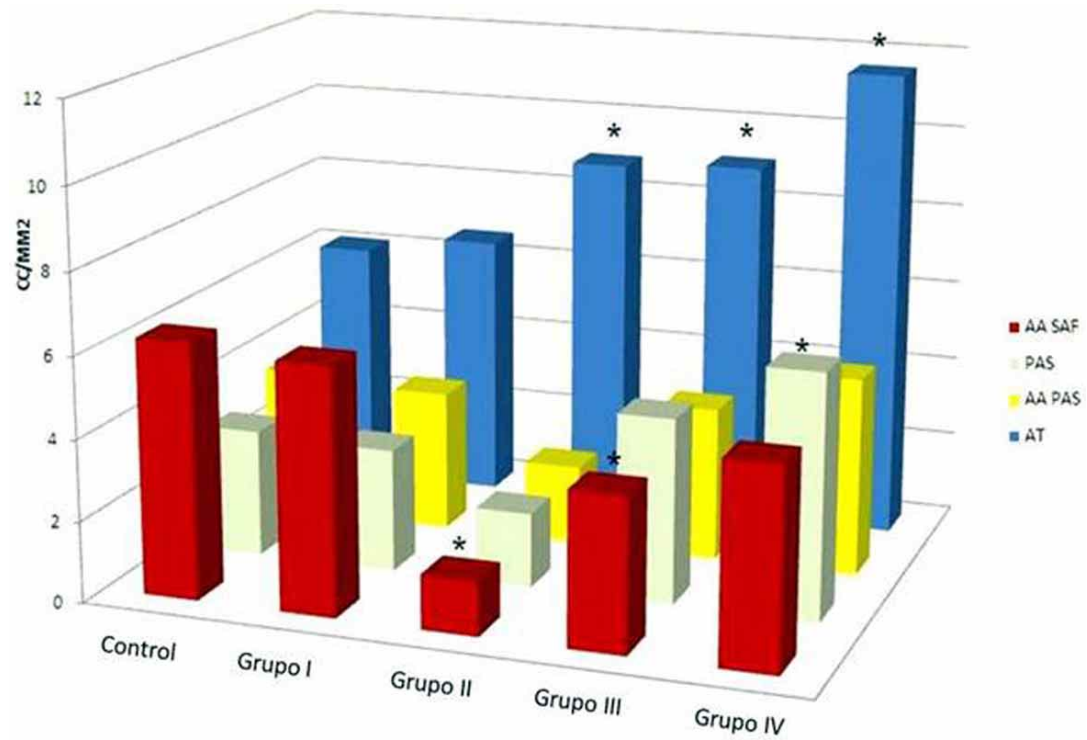

Fig. 2. Grafica que muestra el número de células cebadas en nervio periférico por $\mathrm{mm}^{2}$ (CC/mm2) teñidas con AT y las reacciones histoquímicas de PAS, AA/PAS y AA/SAF en los diferentes grupos de estudio. * Diferencia estadísticamente significativa en relación al control. Prueba t de student. ** Diferencia estadísticamente significativa intergrupos PAS y AAPAS. ANOVA y prueba de Tukey. $\mathrm{P} \leq 0,05$.

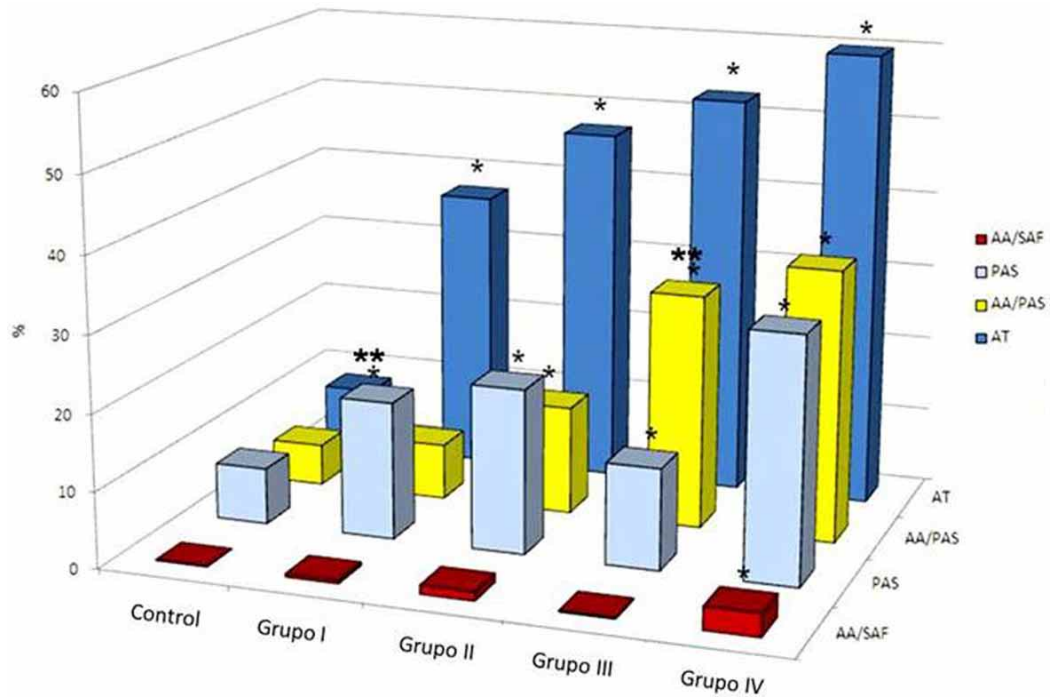

Fig. 3. Grafica que muestra el porcentaje de células cebadas en pulmón teñidas con AT y las reacciones histoquímicas de PAS, AA/PAS y AA/SAF en los diferentes grupos de estudio."Diferencia estadísticamente significativa en relación al control. Prueba t de student. ${ }^{* *}$ Diferencia estadísticamente significativa intergrupos PAS y AA/PAS. ANOVA y prueba de Tukey. P $\leq 0,05$.

Con las pruebas estadísticas de ANOVA determinamos que durante la intoxicación crónica con Kh existen diferencias significativas en cuanto a las varianzas inter-grupos de las células con gránulos positivos para las reacciones de, PAS, AA/PAS y AA/SAF. Con la prueba de Tukey identificamos que estas diferencias en las varianzas inter-grupos dependían principalmente de la escasa cantidad de células cebadas con gránulos AA/SAF positivas en relación a las demás, en todos los grupos experimentales (Grupos I-IV) y entre las PAS y AA/ PAS ya que las células con estos tipos de gránulos presentaron diferencias significativas entre ellos en los grupos II y III (Fig. 3). 
GARCÍA, G. R.; SALAZAR, L. M. E.; BECERRA, V. E. M.; ROMERO, D. V.; GARCÍA, J. J.; SOTO, D. A. \& SEPÚLVEDA, S. J. Células cebadas en pulmón y nervio periférico en la intoxicación crónica con Karwinskia humboldtiana en rata wistar: estudio histológico e histoquímico. Int. J. Morphol., 31(4):1216-1222, 2013.

\section{DISCUSIÓN}

Con base en los resultados obtenidos determinamos que durante la intoxicación crónica con Kh existen modificaciones con respecto al número de células cebadas, en las muestras de nervio periférico y pulmón, en donde hemos demostrado un incremento significativo de las mismas. En el resto de órganos evaluados, las células cebadas fueron escasas y no existieron modificaciones en el número de estas.

Los hallazgos morfológicos en nervio periférico son de gran interés, ya que tanto en el modelo experimental de intoxicación crónica (Salazar-Leal et al.), así como en diversos reportes de casos de intoxicación accidental por Kh en humanos (Bustamante-Sarabia et al.; Puértolas et al.; Segovia \& Zermeño; Arellano et al.) el cuadro paralítico es el dato clínico predominante.

En este estudio hemos descrito por primera vez, los cambios morfológicos presentes en pulmón durante los diferentes grupos del modelo de intoxicación crónica con el fruto maduro de $\mathrm{Kh}$, así como la presencia de grandes acumulos de células cebadas tanto en las regiones peribronquiales como en los tabiques interalveolares de este órgano.

No existen en la literatura reportes sobre el infiltrado de células cebadas, ni sobre la cantidad de células presentes o las variaciones en cuanto a la composición de sus gránulos, en nervio periférico y pulmón durante la intoxicación crónica con Kh, sin embargo, en otros estudios de neuropatías de distintas etiologías, se ha reportado un incremento de estas células en el nervio periférico durante la neuropatía diabética, la esclerosis múltiple, el síndrome de Guillain-Barré, la enfermedad de Alzheimer y la degeneración Waleriana (Bienestock et al.; Olsson \& Sjöstrand, 1969; Esposito et $a l$.), de la misma manera se ha reportado que en el pulmón se presenta un incremento de células cebadas en distintos modelos experimentales de intoxicación química con semillas de Crotoraría spectabilis (Takeoka et al.; Kay et al.), hongos filamentosos (Doebler et al; Jacevic et al.), compuestos del veneno de abeja (Taylor et al.), avispa (Okano et al.), escorpión (De-Matos et al.), entre otros, lo que sugiere que un metabolito o compuesto presente en las plantas de Kh es capaz de inducir dicho incremento.

Tanto en las muestras de nervio periférico como en las de pulmón, se observaron diferencias en cuanto al numero de células cebadas en cada uno de ellos y en los distintos grupos del modelo de intoxicación crónica, además de variaciones en cuanto a la composición de los gránulos de células cebadas evidenciados con las reacciones histoquímicas de PAS, AA/PAS y AA/SAF. Llama la atención que en nervio periférico los gránulos positivos para AA/ SAF del grupo II y que en pulmón los gránulos PAS positivos del grupo III sufren un decremento significativo en relación al grupo anterior, lo que sugiere la liberación de sus mediadores y la probable participación de estos en dichos grupos.

En otros modelos experimentales se ha reportado la existencia de una expresión diferencial de gránulos de células cebadas dependiente de diversos factores como la interleucina 3 (IL 3) y el ligando de c-kit (Gurish et al., 1992), además se ha reportado que otros factores biológicos pueden estimular la liberación de gránulos de estas mismas células (Doebler et al.; Forsythe et al., 2001), en conjunto estos datos nos sugieren que las variaciones en cuanto a la composición de los gránulos de las células cebadas observadas en nuestro estudio, se deban a una probable participación de distintos factores biológicos, que a su vez favorezcan la expresión y liberación de distintos mediadores en cada órgano y en cada grupo del modelo de intoxicación crónica con Kh. De la misma manera nuestros resultados sugieren que las diferencias observadas en cuanto a la expresión y liberación de los mediadores presentes en los gránulos, manifestada por incrementos o decrementos de las células con gránulos positivos a las diversas reacciones histoquímicas, incluso en el grupo IV (Recuperación), se deban a que las células cebadas jueguen distintos roles, ya sea de daño, recuperación o ambos, durante esta intoxicación.

En conclusión, los hallazgos reportados en el presente estudio, sugieren que las células cebadas participan activamente en la fisiopatología de la intoxicación crónica con el fruto maduro de Kh, en el pulmón y nervio periférico.

En nuestro laboratorio continuamos estudiando por métodos inmunohistoquímicos los componentes específicos (mediadores) de los gránulos, con el fin de identificar el papel que juegan estos en cada etapa del modelo de intoxicación crónica con Kh.

GARCÍA, G. R.; SALAZAR, L. M. E.; BECERRA, V. E. M.; ROMERO, D. V.; GARCÍA, J. J.; SOTO, D. A. \& SEPÚLVEDA, S. J. Lung and peripheral nerve mast cells in chronic intoxication with Karwinskia humboldtiana in Wistar rat: histological and histochemical study. Int. J. Morphol., 31(4):12161222, 2013.

SUMMARY: Karwinskia humboldtiana $(\mathrm{Kh})$ is a poisonous shrub causing a number of accidental intoxications in humans. In previous studies in our laboratory, we reported an increased number of mast cells present in peripheral nerve of $\mathrm{Kh}$ 
GARCÍA, G. R.; SALAZAR, L. M. E.; BECERRA, V. E. M.; ROMERO, D. V.; GARCÍA, J. J.; SOTO, D. A. \& SEPÚLVEDA, S. J. Células cebadas en pulmón y nervio periférico en la intoxicación crónica con Karwinskia humboldtiana en rata wistar: estudio histológico e histoquímico. Int. J. Morphol., 31(4):1216-1222, 2013.

intoxicated rats. This finding has not been reported in other organs of intoxicated animals. For this reason, in the present study we searched for mast cells in several organs, identifying mast cell subpopulations on the basis of different histochemical reactivity of their secretory granules. Thus the objective of the present study was to evaluate the presence of mast cells in organs other than peripheral nerve and, to distinguish mast cells by their granule content, applying histochemical reactions. 32 Wistar rats were divided into 4 groups $(n=8)$. For each group, 5 rats were intoxicated with $\mathrm{Kh}$ and 3 received water only as a control. Intoxicated rats received $3.5 \mathrm{~g} / \mathrm{Kg}$ body weight of dry powder of $\mathrm{Kh}$ fruits, fractionated in 5 doses as follows 1.5, 0.5, 0.5, 0.5, 05 on days 0 , $3,7,10,14$ respectively. Control rats received water only. Each group was killed at different times during paralysis evolution. Samples of liver, kidney, lung and brain, were obtained and processed by routine technique until paraffin embedding. Sections were obtained and stained with toluidine blue, PAS, alcian blue/PAS and alcian blue/safranin. Mast cells infiltrates were observed in peripheral nerve and lung. Mast cells were counted. An increasing number of mast cells were recorded as well as variations in the histochemical pattern of their granules for each organ. These findings suggest a role for mast cells and their secretions in the intoxication with mature fruit of $\mathrm{Kh}$.

KEY WORDS: Karwinskia humboldtiana; Mast cells; Peripheral nerve; Lung.

\section{REFERENCIAS BIBLIOGRÁFICAS}

Arellano, E. C.; Mendoza, J. C. \& Domínguez, F. C. Intoxicación por Karwinskia johnstonii: Estudio de 12 pacientes. Bol. Med. Hosp. Infant. Mex.. 51(2):105-12, 1994.

Becerra-Verdin, E.; Bermúdez-Barba, M. V.; Salazar-Leal, M. E.; Ancer Rodríguez, J.; Romero-Diaz, V.; Soto-Domínguez, A.; Ballesteros-Eliozondo, R. G.; Saucedo-Cárdenas, O.; Piñeyro, L. A. \& Sepúlveda-Saavedra, J. Karwinskia humboldtiana (buckthorn) fruit causes central nervous system damage during chronic intoxication in the rat. Toxicon, 53(6):645-51, 2009.

Bermúdez, M. V.; Lozano, F. E.; Salazar, M. E.; Waksman, N. \& Piñeyro, A. Intoxicación de una familia con Karwinskia humboldtiana (tullidora). Gac. Méd. Mex., 131(1):100-6, 1995.

Bienenstock, J.; MacQueen, G.; Sestini, P.; Marshall, J. S.; Stead, R. H. \& Perdue, M. H. Mast cell/nerve interactions in vitro and in vivo. Am. Rev. Respir. Dis., 143(3 Pt.2):S55-8, 1991.

Bustamante-Sarabia, J.; Olvera, J. \& Nieto, L. Intoxicación fatal por tullidora (Karwinskia humboldtiana). Comunicación de un caso. Gac. Méd. Mex., 114:241-4, 1978.

Castillo, F. Contribución al estudio de la parálisis toxica. Un envenenamiento colectivo con tullidora. En: Memorias del V Congreso Médico Mexicano. Puebla, Instituto Médico Nacional, 1920. pp.240-3.
De-Matos, I. M.; Talvani, A.; Rocha, O. O.; Freire-Maia, L. \& Teixeira, M. M. Evidence for a role of mast cells in the lung edema induced by Tityus serrulatus venom in rats. Toxicon, 39(6):863-7, 2001.

Doebler, J. A.; Martin, L. J.; Morse, J. D.; Ballough, G. P.; Strauss, J.A. \& Anthony, A. Mesenteric mast cell degranulation in acute T-2 toxin poisoning. Toxicol. Lett., 62(1):33-8, 1992.

Dreyer, D. L.; Arai, I.; Bachman, C. D.; Anderson, W. R.; Smith, R. \& Daves, D. Toxins Causing no inflammatory Paralityc Neuropathy Isolation and Structure Elutidation. J. Am. Chem. Soc., 97:4895-990, 1975.

Esposito, P.; Chandler, N.; Kandere, K.; Basu, S.; Jacobson, S.; Connolly, R.; Tutor, D. \& Theoharides, T. C. Corticotropinreleasing hormone and brain mast cells regulate blood-brainbarrier permeability induced by acute stress. J. Pharmacol. Exp. Ther., 303(3):1061-6, 2002.

Fernández, N. R. Nombres Comunes uso y distribución del genero Karwinskia (Rhamnaceae) en México. Anales Inst. Biol. Univ. Nac. Autón. México, Ser. Bot., 63(1):1-23, 1992.

Forsythe, P.; Gilchrist, M.; Kulka, M. \& Befus, A. Mast cells and nitric oxide: control of production, mechanisms of response. Int. Immunopharmacol., 1(8):1525-41, 2001.

Gurish, M. F.; Ghildyal, N.; McNeil, H. P.; Austen, K. F.; Gillis, S. $\&$ Stevens, R. L. Differential expression of secretory granule proteases in mouse mast cells exposed to interleukin 3 and ckit ligand. J. Exp. Med., 175(4):1003-12, 1992.

Jacevic, V.; Bokonjic, D.; Stojiljkovic, M.; Resanovic, R.; BocarovStanc`ic', A.; Kilibarda, V. \& Popovic', N. Morphometric changes of cardiac mast cells in rats acutely poisoned by $\mathrm{T}-2$ toxin. Acta. Vet., 57(1):47-57, 2007.

Kay, J. M.; Gillund, T. D. \& Heath, D. Mast cells in the lungs of rats fed on Crotalaria spectabilis seeds. Am. J. Pathol., 51(6):1031-44, 1967.

Metcalfe, D. D.; Baram, D. \& Mekori, Y. A. Mast cells. Physiol. Rev., 77(4):1033-79, 1997.

Ocampo-Roosens, L. V.; Ontiveros-Nevares, P. G. \& FernándezLucio, O. Intoxication with buckthorn (Karwinskia humboldtiana): report of three siblings. Pediatr. Dev. Pathol., 10(1):66-8, 2007.

Okano, Y.; Takagi, H.; Tohmatsu, T.; Nakashima, S.; Kuroda, Y.; Saito, K. \& Nozawa, Y. A wasp venom mastoparan-induced polyphosphoinositide breakdown in rat peritoneal mast cells. FEBS Lett., 188(2):363-6, 1985.

Olsson, Y. \& Sjöstrand, J. Proliferation of mast cells in peripheral nerves during Wallerian degeneration. A radioautographic study. Acta Neuropathol., 13(2):111-21, 1969. 
GARCÍA, G. R.; SALAZAR, L. M. E.; BECERRA, V. E. M.; ROMERO, D. V.; GARCÍA, J. J.; SOTO, D. A. \& SEPÚLVEDA, S. J. Células cebadas en pulmón y nervio periférico en la intoxicación crónica con Karwinskia humboldtiana en rata wistar: estudio histológico e histoquímico. Int. J. Morphol., 31(4):1216-1222, 2013.

Puértolas, M. M. A.; Nava, J. D.; Medina, L. H. A.; López, O. F. X. \& Oyervides, P. J. F. Polirradiculoneuritis por Karwinskia humboldtiana. Informe de seis casos. Rev. Méd. IMSS, 22(1):22-7, 1984.

Salazar-Leal, M. E.; Flores, M. S.; Sepulveda-Saavedra, J.; Romero-Diaz, V. J.; Becerra-Verdin, E. M.; Tamez-Rodriguez, V. A.; Martinez, H. R.; Piñeyro-Lopez, A. \& Bermudez, M. V. An experimental model of peripheral neuropathy induced in rats by Karwinskia humboldtiana (buckthorn) fruit. J. Peripher. Nerv. Syst., 11(3):253-61, 2006.

Segovia, P. A. \& Zermeño, F. Polirradiculoneuritis toxica por Karwinskia humboldtiana. A propósito de un caso. Biol. Med. Hosp. Infant., 29:98-90, 1972.

Takeoka, O.; Angevine, D. M. \& Lalich, J. Stimulation of mast cells in rats fed various chemicals. Am. J. Pathol., 40:545-54, 1962.

Taylor, J. W.; Bidard, J. N. \& Lazdunski, M. The characterization of high-affinity binding sites in rat brain for the mast celldegranulating peptide from bee venom using the purified monoiodinated peptide. J. Biol. Chem., 259(22):13957-67, 1984.

\author{
Dirección para Correspondencia: \\ Dr. Julio Sepúlveda Saavedra \\ Departamento de Histología \\ Facultad de Medicina de la UANL, AP 1563 \\ Monterrey, N.L. \\ MÉXICO
}

Email:jusesaa@netscape.net

Recibido: 29-12-2012

Aceptado: 03-08-2013 